\title{
Advancing Atomic-Resolution TEM of Electron Beam-Sensitive Crystalline Materials from "Impossible" to "Routine"
}

\author{
Daliang Zhang ${ }^{1}$, Lingmei Liu ${ }^{1}$, Yihan $\mathrm{Zhu}^{1}$ and $\mathrm{Yu} \mathrm{Han}^{1 *}$ \\ 1. King Abdullah University of Science and Technology, Thuwal, Saudi Arabia. \\ * Corresponding author: yu.han@kaust.edu.sa
}

High-resolution imaging of electron beam-sensitive crystalline materials is one of the most difficult applications of transmission electron microscopy (TEM). The challenges are manifold, including the acquisition of images with an extremely low beam dose, the time-constrained search for crystal zone axes, the precise alignment of successive images, and the accurate determination of the defocus value.

We reported that using a direct-detection electron-counting (DDEC) camera, it is possible to acquire useful high-resolution TEM images with electron dose as low as a few electrons per square angstrom to ensure that the intact structure was captured before damage occurred [1]. We demonstrated this strategy using a metal organic framework (MOF) material, ZIF-8, as a typical example, as MOFs are generally recognized as highly sensitive to electron beams. In this work, the zone-axis image was obtained by sampling a large number of randomly oriented crystals, which is an inefficient trial-and-error process and cannot guarantee success.

In the following work, we further developed a suite of methods that we recently developed to address how to achieve fast seeking of zone axis to avoid beam damage in this process as well as the rest challenges mentioned above. Our methods advance the HRTEM of extremely beam-sensitive materials from "occasionally possible" to "routine", and further push the resolution limit by fully retrieving the high-resolution information hidden in the image stack. We demonstrated the effectiveness of our methodology by capturing atomic-resolution $(\sim 1.5 \AA)$ TEM images of several MOF materials. In the case of MOF UiO-66, individual metal atomic columns, various types of surface termination, and benzene rings in the organic linkers, have been clearly identified [2]. We have successfully applied our methods to other electron beam-sensitive materials, including covalent organic frameworks (COFs), two-dimensional supramolecular crystals, and crystals constructed by self-assembly of metal cluster, and achieved atomic-resolution TEM imaging of the organic-inorganic hybrid perovskite $\mathrm{CH}_{3} \mathrm{NH}_{3} \mathrm{PbBr}_{3}$ for the first time [2].

More recently, we applied this new technology to directly observe encapsulated single molecule magnets in MOF NU-1000 [3] (Figure 1), and to investigate the evolution and transformation of various defects in MOF UiO-66 [4]. We identified three types of structural defects, two of which were completely unknown in the MOF community because they are essentially invisible by other characterization techniques but HRTEM. The high-quality images acquired from different zone axes allowed us to three-dimensionally reconstructed the "missing linker" defect with extraordinary precision to reveal the defect-terminating formate groups (Figure 2).

The advent of low-dose HRTEM heralds a new way to explore the link between the structure of electron-sensitive materials and their functionalities through unambiguous structure determination. 
References:

[1] Y Zhu et al., Nature Materials 16 (2017), p. 532.

[2] D Zhang et al., Science 359 (2018), p. 675.

[3] M Wriedt et al., J. Am. Chem. Soc. (2019) (accepted).

[4] L Liu et al., Nature Chemistry (2019) (accepted).
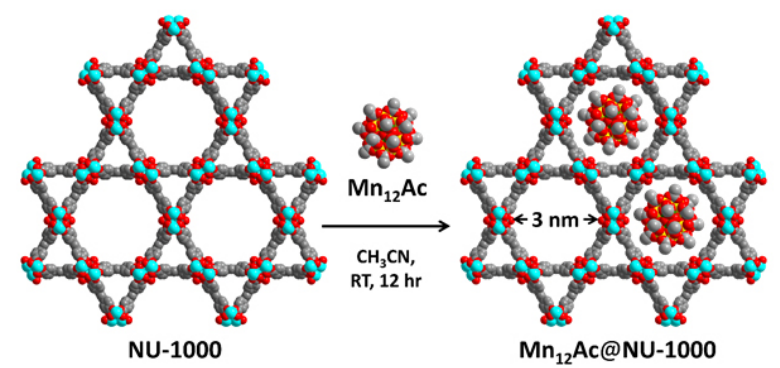
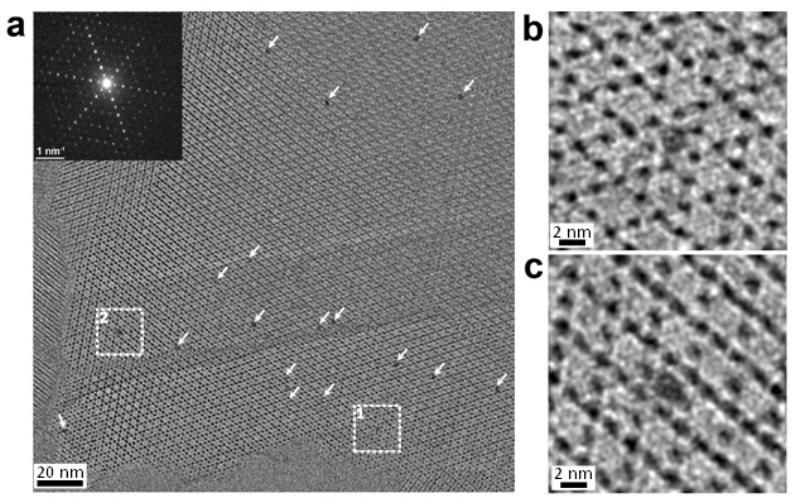

Figure 1. (left) Schematic illustration of the encapsulation of single-molecule magnets $\left(\mathrm{Mn}_{12} \mathrm{Ac}\right.$ clusters) into the channels of MOF NU-1000. (a) HRTEM image and electron diffraction pattern (inset) of $\mathrm{Mn}_{12} \mathrm{Ac} @ \mathrm{NU}-1000$ acquired along the [001] zone axis. Arrows indicate the observed single-molecule magnet clusters. (b) and (c) Enlarged images of the highlighted areas in (a), showing that the clusters are precisely encapsulated and perfectly fitted in the hexagonal channels of NU-1000.

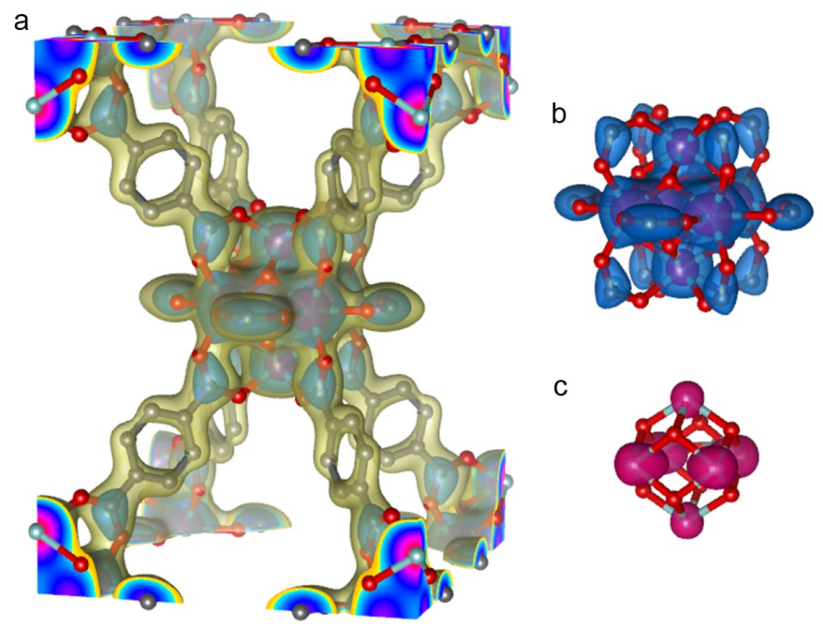

Figure 2. Reconstructed electron potential map of the "missing linker" defect in MOF UiO-66 from HRTEM images. Isosurfaces rendered at different thresholds are superimposed with the geometryoptimized structure model of the missing linker defect for comparison. Clearly, the envelope of the defective UiO-66 framework, which is composed of $\mathrm{Zr}$ cluster, BDC linkers and defect-terminating formate groups, is identified at the threshold of $48.5 \%$ (a). The $\mathrm{C}$ atoms on carboxyl groups and the $\mathrm{Zr}$ atoms are precisely identified at the threshold of $56.0 \%$ (b) and $74.5 \%$ (c), respectively. 